\title{
Clinical manifestations of invasive pneumococcal disease by vaccine and non-vaccine types
}

\author{
Sarah Browall ${ }^{1,12}$, Erik Backhaus 2,12 , Pontus Naucler ${ }^{1,3}$, Ilias Galanis ${ }^{1}$, \\ Karin Sjöström ${ }^{1}$, Diana Karlsson ${ }^{4}$, Stefan Berg ${ }^{5}$, Joachim Luthander ${ }^{6}$, \\ Margareta Eriksson ${ }^{6}$, Carl Spindler ${ }^{3}$, Mikael Ejdebäck $^{4}$, Birger Trollfors ${ }^{5}$, \\ Jessica Darenberg ${ }^{7}$, Mats Kalin ${ }^{3}, \AA^{\circ}$ e Örtqvist ${ }^{8,9}$, Rune Andersson ${ }^{10}$ and \\ Birgitta Henriques-Normark ${ }^{1,11}$
}

\begin{abstract}
Affiliations: ${ }^{1}$ Dept of Microbiology, Tumor and Cell Biology, Karolinska Institutet, Stockholm, Sweden. ${ }^{2}$ Dept of Infectious Diseases, Skaraborg Hospital, Skövde, Sweden. ${ }^{3}$ Dept of Infectious Diseases, Karolinska University Hospital, Solna, Sweden. ${ }^{4}$ Systems Biology Research Centre, School of Biosciences, University of Skövde, Skövde, Sweden. ${ }^{5}$ Queen Silvia Children's Hospital, Sahlgrenska University Hospital, Gothenburg. Sweden. ${ }^{6}$ Dept of Paediatrics, Karolinska University Hospital, Solna, Sweden. ${ }^{7}$ Public Health Agency of Sweden, Solna, Sweden. ${ }^{8}$ Dept of Communicable Diseases Control and Prevention, Stockholm County Council, Stockholm, Sweden. ${ }^{9}$ Dept of Medicine, Unit of Infectious Diseases, Karolinska Institutet, Solna, Sweden. ${ }^{10}$ Dept of Infectious Diseases, Institute of Biomedicine, Sahlgrenska Academy, Gothenburg University, Gothenburg, Sweden. ${ }^{11}$ Dept of Clinical Microbiology, Karolinska University Hospital, Stockholm, Sweden. ${ }^{12}$ Both authors contributed equally.
\end{abstract}

Correspondence: Birgitta Henriques-Normark, Dept of Microbiology, Tumor and Cell Biology, Karolinska Institutet, Nobelsväg 16, 17177 Stockholm, Sweden. E-mail: birgitta.henriquesaki.se

ABSTRACT Pneumococcal conjugated vaccines (PCVs) have shown protection against invasive pneumococcal disease by vaccine serotypes, but an increase in non-vaccine serotype disease has been observed. Type-specific effects on clinical manifestation need to be explored.

Clinical data from 2096 adults and 192 children with invasive pneumococcal disease were correlated to pneumococcal molecular serotypes. Invasive disease potential for pneumococcal serotypes were calculated using 165 invasive and 550 carriage isolates from children.

The invasive disease potential was lower for non-PCV13 compared to vaccine-type strains. Patients infected with non-PCV13 strains had more underlying diseases, were less likely to have pneumonia and, in adults, tended to have a higher mortality. Furthermore, patients infected with pneumococci belonging to clonal serotypes only expressing non-PCV13 capsules had a higher risk for septicaemia and mortality.

PCV vaccination will probably lead to a decrease in invasive pneumococcal disease but an alteration in the clinical manifestation of invasive pneumococcal disease. Genetic lineages causing invasive pneumococcal disease in adults often express non-vaccine serotypes, which can expand after vaccination with an increased risk of infection in patients with underlying diseases.

@ERSpublications

Pneumococcal non-vaccine type strains cause severe disease, but with different spectrums of clinical manifestations http://ow.ly/ABYnz

This article has supplementary material available from erj.ersjournals.com

Received: May 012014 | Accepted after revision: Aug 082014 | First published online: Oct 162014

Support statement: This work was supported by research grants from the Swedish Research Council (Stockholm, Sweden), the Knut and Alice Wallenberg Foundation (Stockholm), the Swedish Foundation for Strategic Research (Stockholm), Torsten and Ragnar Söderbergs Foundation (Stockholm), the Swedish Royal Academy of Sciences (Stockholm), the Swedish Knowledge Foundation (Stockholm), ALF-grant from Stockholm City Council (Stockholm), the research fund at Skaraborg Hospital (Skövde, Sweden), the Health and Medical Care Executive Board of Region Västra Götaland and Göteborg University (Gothenburg, Sweden).

Conflict of interest: Disclosures can be found alongside the online version of this article at erj.ersjournals.com

Copyright (CERS 2014 ERJ Open articles are open access and distributed under the terms of the Creative Commons Attribution Non-Commercial Licence 4.0. 


\section{Introduction}

Streptococcus pneumoniae or pneumococci are major causes of morbidity and mortality worldwide, especially in small children and the elderly [1]. Pneumococci express over 90 capsular serotypes, which have different potential to cause disease [2-5]. Pneumococcal conjugate vaccines (PCVs) have been introduced into the childhood vaccination programme in many countries, including Sweden. The serotypes included were chosen based on their abundance in children with invasive pneumococcal disease. PCV7, including the paediatric serotypes $4,6 \mathrm{~B}, 9 \mathrm{~V}, 14,18 \mathrm{C}, 19 \mathrm{~F}$ and $23 \mathrm{~F}$, was introduced first and has shown good protection in most studies [6-12]. The additional three serotypes (1, 5 and 7F) included in PCV10 provide protection against strains frequently associated with pneumonia [13], and the additional serotypes in PCV13 are expected to protect against the common paediatric serotype 6A and serotype 19A, which was observed to expand early after PCV7 introduction $[14,15]$. It is still not clear whether PCV13 will provide protection against serotype 3, an important serotype associated with high mortality $[2,3,16]$.

The ecological niche for pneumococci is the nasopharynx of healthy preschool children from where the bacteria may spread and cause disease in other age groups. Due to herd immunity, vaccine type strains have also been observed to decrease as causes of invasive pneumococcal disease in non-vaccinated adults $[8,17]$. Recently, in the USA, it was also shown that the overall incidence of pneumonia has been reduced in adults after the introduction of PCV7 [18]. However, PCVs select for non-vaccine type strains, and replacement of vaccine type with non-vaccine type strains in the nasopharynx of vaccinated children has led to a completely changed spectrum of pneumococcal serotypes circulating in the community, which also affects invasive pneumococcal disease in non-vaccinated adults. The extent of type replacement differs between populations and in Stockholm County in Sweden, to date, no net reduction of invasive pneumococcal disease in older adults has been observed due to an increase of serotypes not included in the PCV13 vaccine (data not shown) [19]. Some studies have investigated the potential association of serotype to clinical disease [2, 20,21]; however, further data is needed to understand to what extent non-vaccine strains provide the same ability to cause invasive pneumococcal disease and whether the disease manifestations are the same as for vaccine strains. Furthermore, pneumococci are genetically diverse due to an efficient DNA transformation system and molecular typing regimes can distinguish a number of genetically related clonal lineages that might express different capsular serotypes $[2,3,16]$. It is not clear to what extent invasiveness, disease manifestation and severity correlate to other properties reflected by the clonal type, such as virulence genes [22].

The introduction of PCV has resulted in the expansion of pre-existing non-vaccine type strains. Hence, it is possible to use large pre-vaccination materials to predict the expected invasiveness and clinical disease manifestations of non-vaccine replacement strains, which might influence the clinical management, development and selection of vaccines. Herein, we used one of the largest available collections of patient data and corresponding pneumococcal isolates from invasive pneumococcal disease in adults and children in Sweden, as well as nasopharyngeal isolates from healthy children collected before the introduction of vaccines. The aim was to calculate the invasive disease potential for different serotypes and explore possible correlations of clinical manifestations and disease outcome of vaccine and non-vaccine serotypes and clonal serotypes.

\section{Materials and methods}

\section{Patients and pneumococcal isolates}

To calculate the odds ratio for causing invasive disease, 550 nasopharyngeal carriage isolates (collected from children attending day care centres) and 165 invasive isolates from children $(<18$ years of age) from the Stockholm area collected between 1997 and 2004 prior to vaccination were included [22, 23].

To correlate clinical parameters to strain characteristics invasive pneumococcal disease isolates from blood, cerebrospinal fluid and other normally sterile sites were collected from clinical microbiological laboratories in Sweden from 1998 to 2008 for 2096 adults and 192 children aged < 18 years, mainly during the pre-vaccination period (PCV7 was introduced into the child immunisation programme in a few Swedish counties at the end of 2007 and the rest of Sweden in 2009). Clinical data were collected for the patients (1191 patients were included in previous studies) [2, 21, 24]. Only the first event in each patient during the study period was included in the analyses. This was done to avoid inclusion of re-infections caused by the same strain. Ethical approval was obtained from the regional ethical committees in Stockholm, Lund and Gothenburg (all Sweden).

\section{Serotyping and molecular typing}

Serotyping using antisera directed against the capsular polysaccharide was performed according to standard methods as described previously $[3,21]$. To further study the genetic relatedness between the 
strains, molecular typing was performed to identify different genetic clonal types. All isolates were characterised using pulsed-field gel electrophoresis (PFGE) as adapted from Hermans et al. [25], where pneumococcal chromosomal DNA was cleaved by the restriction enzyme Apa I and the DNA fragments were separated using PFGE. Multi-locus sequence typing (MLST) (where parts of the house-keeping genes aroE, $g d h, g k i$, recP, spi, xpt and $d d l$ within the pneumococcal genome were sequenced) was performed on selected strains from each dominant PFGE pattern; 511 isolates in total [26]. Based on MLST results, isolates in the same PFGE clone were considered to belong to the same clonal complex.

\section{Statistical analyses}

Odds ratios with 95\% confidence interval were calculated to study the invasive disease potential of serotypes. The odds ratio was calculated using the formula:

$$
\text { odds ratio }=(a d) /(b c)
$$

where $a$ was the number of invasive serotype $\mathrm{X}, b$ the number of carriage serotype $\mathrm{X}, c$ the number of invasive serotype non-X and $d$ the number of carriage serotype non-X. Normal approximation was used to calculate the confidence intervals and the respective p-values. Fisher's exact test was implemented when appropriate. Holm's method was applied to adjust the p-values for multiple comparisons [27].

Odds ratios and their respective $95 \%$ confidence intervals were calculated to estimate differences between vaccine serotype groups and clinical parameters. Pair-wise calculations were performed for all serotype group combinations. Fisher's exact test and Holm's method were applied as described previously.

Logistic regression models were fitted to assess the association between individual clonal complexes (using CC124 as a reference) or serotypes (using serotype 14 as a reference) with disease manifestation, underlying diseases, 30-day mortality (for adults) and intensive care unit stay for $>24 \mathrm{~h}$ (for children) as outcome variables. The models on disease manifestation and 30-day mortality for adults were adjusted for age and comorbidity using the Charlson index score, while underlying diseases was only adjusted for age. The penalised likelihood logistic regression of FIRTH [28] was performed for children, in order to deal with separation problems and small sample bias with no adjustment for other covariates. Results are presented from those models where the clonal complex variable was significant after ANOVA. The significance level was set at $5 \%$ in all analyses. Computations were performed using the statistical software $\mathrm{R}$ (version 3.0.1; http://www.r-project.org).

\section{Results}

Invasive disease potential was lower for non-PCV13 strains compared to vaccine type strains

The invasive disease potential (odds ratio) for vaccine type and non-vaccine type strains was calculated (table 1). The odds ratio was significantly higher for serotype 19A, which is only present in PCV13, as well as for serotypes 1 and 7F, both of which are absent in PCV7 but present in PCV10. Hence, the odds ratio for causing invasive disease for the extra serotypes present in PCV10 was higher than for serotypes present in PCV7 only (tables 1 and 2). Importantly, non-PCV13 strains had a significantly lower odds ratio for causing invasive disease in children when compared to serotypes present in PCV7, PCV10 and PCV13 (table 2).

\section{Infections with non-PCV13 strains seldom manifest as pneumonia and often affect children with underlying diseases}

To estimate the relationships between pneumococcal types, disease manifestation and underlying diseases among children, isolates and corresponding clinical data from 192 children were included. The mean age was 4 years (median age 2 years) and the children were predominately male (63\%). 140 (73\%) children had either pneumonia or meningitis while the remaining children mainly suffered from septicaemia with an unknown focus. A broad range of underlying diseases were present in $26 \%$ of the children, with congenital disorders, heart disease and leukaemia being the most common (table 3 and fig. 1a). Four (2\%) children died, three of whom had underlying diseases. Infections with PCV7 strains had a lower odds ratio for causing pneumonia compared to the additional serotypes in PCV10, but tended to have a higher odds ratio for meningitis (not significant after adjustment for multiple comparisons) (table 3). In addition, previous underlying diseases tended to be more frequently associated with PCV7 strains compared to those with the three extra serotypes in PCV10. The extra three serotypes uniquely present in PCV13 gave similar disease manifestations as PCV7 serotypes. The 15 children infected with non-PCV13 strains often had more underlying diseases as compared to those infected with PCV7, PCV10 or PCV13 strains, and pneumonia as disease manifestation was uncommon (two (13\%) out of 15). 
TABLE 1 Odds ratios for causing invasive disease for different serotypes in children from Stockholm, Sweden

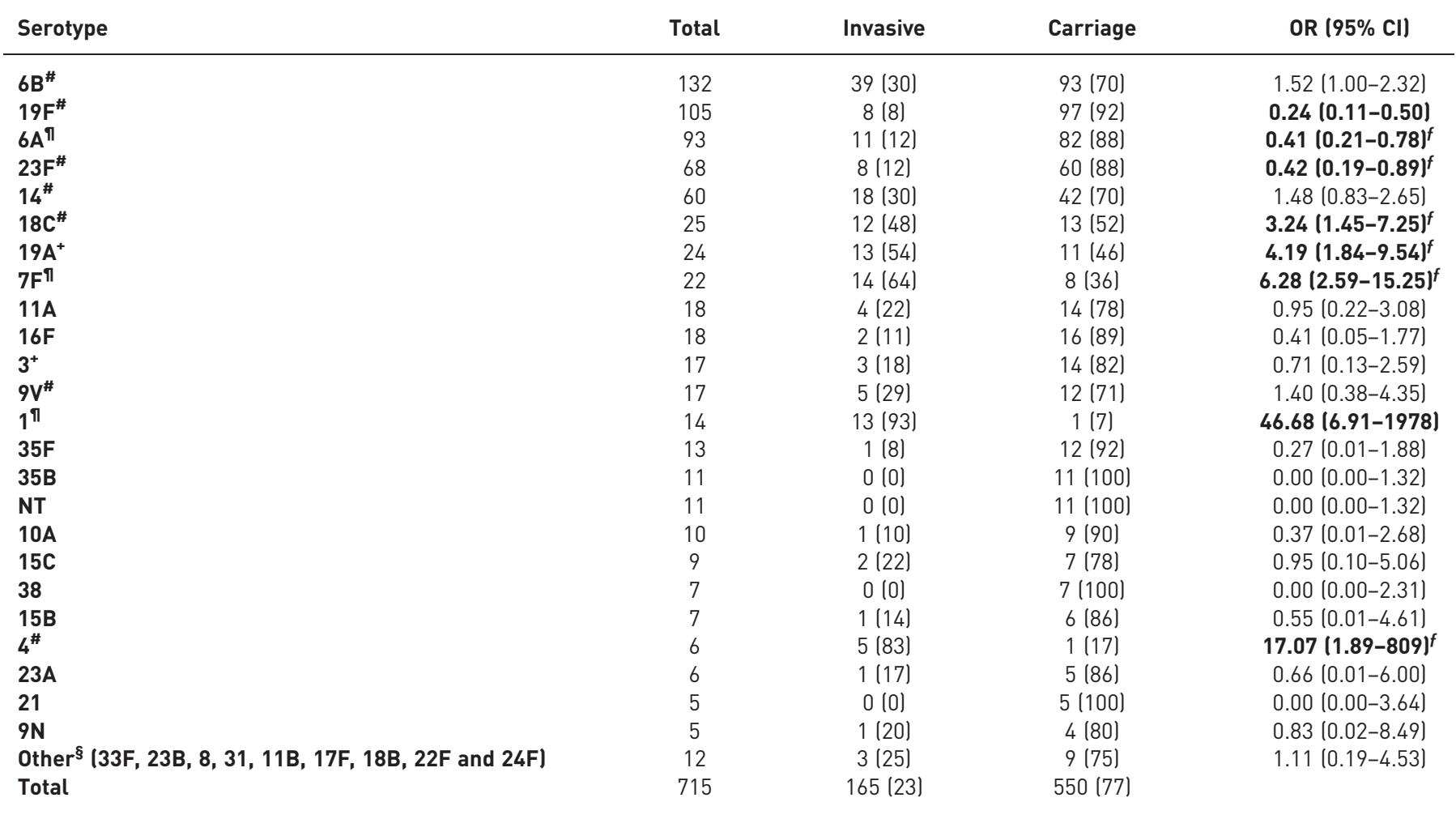

Data are presented as $\mathrm{n}$ or $\mathrm{n}(\%)$, unless otherwise stated. Serotypes representing five or more isolates are shown. Serotypes with an odds ratio $>1$ have an increased risk for causing invasive disease. Bold indicates statistical significance. \#: serotypes included in the 7-valent vaccine; ": additional serotypes included in the 10 -valent vaccine; ${ }^{+}$: additional serotypes included in the 13 -valent vaccine; ${ }^{\S}$ : less than five isolates, all were included in the non-10-valent vaccine; ${ }^{f}$ : not significant after adjustment for multiple comparisons (Holm's method).

\section{Infections with non-PCV13 strains associated with changes in clinical manifestations and increased mortality in adults}

Vaccine serotypes used in the childhood vaccination programme will, due to herd immunity, probably also decrease invasive pneumococcal disease in adults. However, we expect an increased disease burden of non-vaccine types, and most of these types already existed in the population prior to vaccine introduction.

TABLE 2 Odds ratios for causing invasive pneumococcal disease in children in Stockholm, Sweden, among serotypes included or not included in the conjugated vaccines 17, 10 - or 13-valent vaccines)

\begin{tabular}{|c|c|c|c|}
\hline Serotype group & Invasive & Carriage & OR $(95 \% \mathrm{CI})^{\#}$ \\
\hline Serotypes in the 7 -valent vaccine & $95(23)$ & $318(77)$ & $0.10(0.05-0.22)^{\pi}$ \\
\hline $1,5,7 \mathrm{~F}$ (extra in 10 -valent) & $27(75)$ & 9 (25) & 1 (Ref) \\
\hline $3,6 \mathrm{~A}, 19 \mathrm{~A}$ (extra in 13 -valent) & $27(20)$ & $107(80)$ & 1 (Ref) \\
\hline Serotypes in the 7-valent vaccine & $95(23)$ & $318(77)$ & $2.17(1.22-3.83)^{\pi}$ \\
\hline Non-13-valent vaccine serotypes & $16(12)$ & $116(88)$ & 1 (Ref) \\
\hline $3,6 \mathrm{~A}, 19 \mathrm{~A}$ (extra in 13 -valent) & $27(20)$ & $107(80)$ & $1.83(0.93-3.58)$ \\
\hline Non-13-valent vaccine serotypes & $16(12)$ & $116(88)$ & 1 (Ref) \\
\hline Serotypes in the 13 -valent vaccine & $149(26)$ & $434(74)$ & $2.49(1.43-4.34)^{\pi}$ \\
\hline Non-13-valent vaccine serotypes & $16(12)$ & $116(88)$ & 1 (Ref) \\
\hline Total & 165 (23) & $550(77)$ & \\
\hline
\end{tabular}

Data are presented as $\mathrm{n}(\%)$, unless otherwise stated. Bold indicates statistical significance. Ref: reference. ${ }^{\#}$ : crude odds ratio; ${ }^{\text {?: }}$ remained significant after adjustment for multiple comparisons (Holm's method). 
TABLE 3 Odds ratio for disease manifestation in children in Sweden among serotypes included or not included in the conjugated vaccines (7-, 10- or 13-valent vaccines)

\begin{tabular}{|c|c|c|c|c|c|c|c|c|c|c|c|c|c|c|}
\hline \multirow[t]{2}{*}{ Serotype group } & \multirow{2}{*}{$\begin{array}{c}\text { Patients } \\
\text { n }\end{array}$} & \multirow{2}{*}{$\begin{array}{c}\text { Mean age } \\
\text { years }\end{array}$} & \multicolumn{2}{|c|}{ Pneumonia } & \multicolumn{2}{|c|}{ Meningitis } & \multicolumn{2}{|c|}{ Septicaemia } & \multicolumn{2}{|c|}{ Underlying disease } & \multicolumn{2}{|c|}{ ICU >24 h } & \multicolumn{2}{|c|}{ Mortality } \\
\hline & & & $n(\%)$ & $\begin{array}{l}\text { Crude OR } \\
(95 \% \mathrm{Cl})\end{array}$ & $n(\%)$ & $\begin{array}{l}\text { Crude OR } \\
(95 \% \mathrm{Cl})\end{array}$ & $n(\%)$ & $\begin{array}{l}\text { Crude OR } \\
(95 \% \mathrm{Cl})\end{array}$ & $n(\%)$ & $\begin{array}{l}\text { Crude OR } \\
(95 \% \mathrm{Cl})\end{array}$ & $n(\%)$ & $\begin{array}{l}\text { Crude OR } \\
(95 \% \mathrm{Cl})\end{array}$ & $n(\%)$ & $\begin{array}{l}\text { Crude OR } \\
(95 \% \mathrm{Cl})\end{array}$ \\
\hline 7-valent vaccine & 101 & 3 & 33 (33) & $0.21(0.1-0.43)$ & $36(36)$ & $3.02(1.28-7.13)^{\#}$ & $17(17)$ & $1.88(0.61-6.94)$ & 27 (27) & $2.74(1.05-7.14)^{\#}$ & 30 (31) & $3.94(1.37-13.98)$ & $2(2)$ & $\infty(0.09-\infty)$ \\
\hline 1, 5, $7 \mathrm{~F}$ (10-valent) & 51 & 6 & $36(71)$ & 1 (Ref) & $8(16)$ & 1 (Ref) & $5(10)$ & 1 (Ref) & $6(12)$ & 1 (Ref) & $5(10)$ & 1 (Ref) & $0(0)$ & 1 (Ref) \\
\hline 7-valent vaccine & 101 & 3 & 33 (33) & $0.88(0.35-2.19)$ & $36(36)$ & $1.00(0.40-2.49)$ & $17(17)$ & $0.65(0.23-1.86)$ & 27 (27) & $1.16(0.42-3.20)$ & $30(31)$ & $1.76(0.57-6.56)$ & 2 (2) & $0.49(0.02-29.78)$ \\
\hline 3, 6A, 19A (13-valent) & 25 & 2 & $9(36)$ & 1 (Ref) & $9(36)$ & 1 (Ref) & $6(24)$ & 1 (Ref) & $6(24)$ & 1 (Ref) & $5(20)$ & 1 (Ref) & $1(4)$ & 1 (Ref) \\
\hline 7-valent vaccine & 101 & 3 & 33 (33) & $3.18(0.66-30.62)$ & $36(36)$ & $0.64(0.22-1.92)$ & $17(17)$ & $0.41(0.11-1.74)$ & 27 (27) & $0.19(0.05-0.66)$ & 30 (31) & $0.88(0.25-3.59)$ & 2 (2) & $0.29(0.01-17.87)$ \\
\hline Non-13-valent vaccine & 15 & 4 & $2(13)$ & 1 (Ref) & $7(47)$ & 1 (Ref) & $5(33)$ & 1 (Ref) & $10(67)$ & 1 (Ref) & $5(33)$ & 1 (Ref) & 1 (7) & 1 (Ref) \\
\hline 1, 5, $7 \mathrm{~F}$ (10-valent) & 51 & 6 & $36(71)$ & $14.90(2.86-151.8)$ & $8(16)$ & $0.21(0.06-0.75)^{\#}$ & $5(10)$ & $0.22(0.04-1.17)$ & $6(12)$ & $0.07(0.01-0.31)$ & $5(10)$ & $0.23(0.04-1.20)$ & $0(0)$ & $0.00(0.00-11.47)$ \\
\hline Non-13-valent vaccine & 15 & 4 & $2(13)$ & 1 (Ref) & $7(47)$ & 1 (Ref) & 5 (33) & 1 (Ref) & $10(67)$ & 1 (Ref) & $5(33)$ & 1 (Ref) & 1 (7) & 1 (Ref) \\
\hline 3, 6A, 19A (13-valent) & 25 & 2 & $9(36)$ & $3.55(0.58-39.45)$ & $9(36)$ & $0.64(0.17-2.36)$ & $6(24)$ & $0.64(0.12-3.36)$ & $6(24)$ & $0.17(0.03-0.78)^{\#}$ & $5(20)$ & $0.51(0.09-2.78)$ & $1(4)$ & $0.59(0.01-49.03)$ \\
\hline Non-13-valent vaccine & 15 & 4 & $2(13)$ & 1 (Ref) & $7(47)$ & 1 (Ref) & $5(33)$ & 1 (Ref) & $10(67)$ & 1 (Ref) & $5(33)$ & 1 (Ref) & $1(7)$ & 1 (Ref) \\
\hline 13-valent vaccine & 177 & 4 & $78(44)$ & $5.14(1.11-48.24)^{\#}$ & $53(30)$ & $0.49(0.17-1.43)$ & $28(16)$ & $0.38(0.11-1.53)$ & $39(22)$ & $0.14(0.04-0.49)$ & $40(23)$ & $0.60(0.18-2.38)$ & $3(2)$ & $0.24(0.02-13.57)$ \\
\hline Non-13-valent vaccine & 15 & 4 & $2(13)$ & 1 (Ref) & $7(47)$ & 1 (Ref) & $5(33)$ & 1 (Ref) & $10(67)$ & 1 (Ref) & $5(33)$ & 1 (Ref) & $1(7)$ & 1 (Ref) \\
\hline Total & 192 & 4 & $80(42)$ & & 60 (31) & & $33(17)$ & & $49(26)$ & & $45(24)$ & & $4(2)$ & \\
\hline
\end{tabular}

Bold indicates statistical significance. ICU: intensive care unit; Ref: reference. ${ }^{\#}$ : not significant after adjustment for multiple comparisons (Holm's method). 

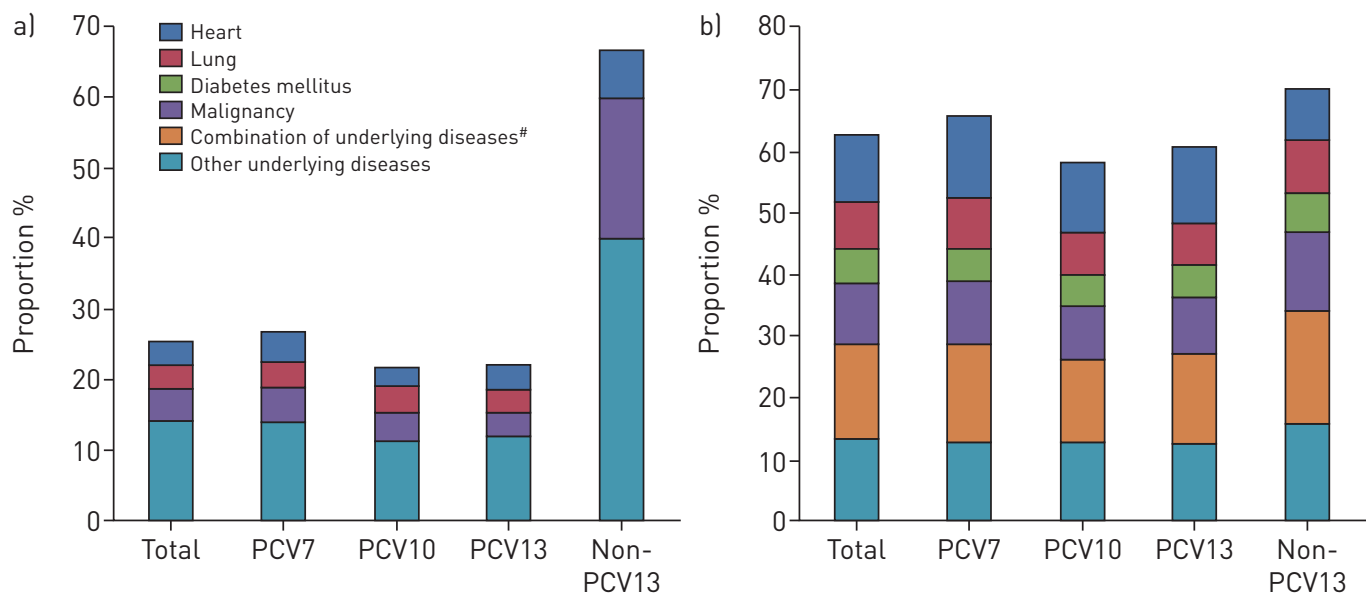

FIGURE 1 Underlying diseases present among a) 192 children and b) 2096 adults. PCV: pneumococcal conjugated vaccines. " : heart, lung, diabetes mellitus and malignancy.

To accurately assess the outcome of invasive pneumococcal disease among adults we included 2096 patients, mainly from the pre-vaccination era. The mean age was 63 years (median age 65 years) and no sex differences were seen ( $49 \%$ females). Pneumonia was the most common manifestation, followed by septicaemia without an identified focus and meningitis (table 4). The overall 30 -day mortality was $11 \%$. Of the patients with septicaemia, meningitis and pneumonia, $22 \%, 18 \%$ and $9 \%$ died, respectively. $63 \%$ had an underlying disease where cardiovascular diseases dominated followed by chronic lung disease, diabetes mellitus, haematological malignancies, solid tumours and alcoholism (fig. 1b).

In total, $484(23.1 \%)$ out of 2096 adults were infected with non-PCV13 serotypes and these patients had a reduced risk of pneumonia, an increased risk of septicaemia with an unknown focus, an increased risk of having underlying diseases and a tendency of increased risk of a fatal outcome compared to patients infected with PCV13 types (table 4). Patients with PCV7 isolates tended to be older, had a significantly lower odds ratio for pneumonia, a significantly higher odds ratio for septicaemia and underlying diseases, and a higher mortality compared to the patients infected with the three extra serotypes included in PCV10. In contrast, PCV7 infected patients had underlying diseases less often and lower mortality compared to patients infected with strains expressing the three extra serotypes 3, 6A and 19A included in PCV13. Interestingly, adults infected with PCV7 strains had a significantly higher level of pneumonia and lower odds ratio of septicaemia compared to patients infected with non-PCV13 strains. In addition, the three serotypes added to PCV10 were associated with a higher odds ratio for pneumonia, a lower odds ratio of septicaemia and lower mortality compared to non-PCV13 types in adults.

Disease manifestation and mortality data for individual serotypes present in at least 20 patients are shown in table S1. Odds ratios were calculated using serotype 14 as a reference, which is the most common serotype in this material. A number of non-PCV13 serotypes $(9 \mathrm{~N}, 12 \mathrm{~F}, 22 \mathrm{~F}$ and $23 \mathrm{~A}$ ) showed a significantly lower odds ratio for pneumonia and increased odds ratio for septicaemia with unknown focus compared to serotype 14. An increased mortality risk was observed in patients infected with the non-PCV13 serotype 11A, as well as $6 \mathrm{~A}$ and 19A, which were included in PCV13, but not in PCV10. Importantly, 162 patients infected with less common serotypes, all non-PCV13, showed a significantly higher odds ratio for septicaemia and had more underlying diseases compared to individuals infected with serotype 14 strains (table S1).

\section{Associations found between pneumococcal clonal types, clinical manifestations and mortality in children and adults}

Since pneumococcal strains of a given serotype may belong to more than one genetically related clonal complex, and since strains belonging to the same clonal complex may express different vaccine or non-vaccine capsular types, we correlated clinical manifestations to clonal types (tables 5 and 6). In children, the most prevalent clonal complexes $(138,191,306,124,9$ and 156) only expressed serotypes present in PCV13, suggesting that they would be eliminated or dramatically decreased in a PCV13 vaccinated region. Other clonal complexes (199 and 113) incorporated both PCV13 and non-PCV13 types, and some clonal complexes (53,460, 30, 433, 218, 414, 72 and 3357) expressed only non-PCV13 serotypes. Children infected with CC306, including only serotype 1 isolates, had a higher odds ratio for having pneumonia and lower odds ratio for meningitis, while those infected with CC138 (serotype 6B and 4) 
TABLE 4 Odds ratios for disease manifestation in adults in Sweden among serotypes included or not included in the conjugated vaccines (7-, 10- or 13-valent vaccines)

\begin{tabular}{|c|c|c|c|c|c|c|c|c|c|c|c|c|}
\hline \multirow[t]{2}{*}{ Serotype group } & \multirow[t]{2}{*}{ Patients n } & \multirow{2}{*}{$\begin{array}{c}\text { Mean age } \\
\text { years }\end{array}$} & \multicolumn{2}{|c|}{ Pneumonia } & \multicolumn{2}{|r|}{ Meningitis } & \multicolumn{2}{|c|}{ Septicaemia } & \multicolumn{2}{|c|}{ Underlying disease } & \multicolumn{2}{|r|}{ Mortality } \\
\hline & & & n (\%) & $\begin{array}{c}\text { Crude OR } \\
(95 \% \mathrm{CI})\end{array}$ & n (\%) & $\begin{array}{c}\text { Crude OR } \\
(95 \% \mathrm{CI})\end{array}$ & n (\%) & $\begin{array}{l}\text { Crude OR } \\
(95 \% \mathrm{Cl})\end{array}$ & $\mathrm{n}(\%)$ & $\begin{array}{l}\text { Crude OR } \\
(95 \% \mathrm{CI})\end{array}$ & $\mathrm{n}(\%)$ & $\begin{array}{c}\text { Crude OR } \\
(95 \% \mathrm{CI})\end{array}$ \\
\hline 7-valent vaccine & 988 & 64 & 806 (82) & $0.52(0.36-0.76)$ & $64(6)$ & $1.82(0.99-3.34)$ & $100(10)$ & $1.98(1.20-3.29)$ & $651(66)$ & $3.25(2.52-4.18)$ & $106(11)$ & $3.43(1.86-6.30)$ \\
\hline $1,5,7 F$ (10-valent) & 354 & 53 & $316(90)$ & 1 (Ref) & $13(4)$ & 1 (Ref) & $19(5)$ & 1 (Ref) & $132(37)$ & 1 (Ref) & $12(3)$ & 1 (Ref) \\
\hline 7 -valent vaccine & 988 & 64 & 806 (82) & $0.87(0.61-1.25)$ & $64(6)$ & $0.87(0.52-1.46)$ & $100(10)$ & $1.27(0.79-2.06)$ & $651(66)$ & $0.69(0.51-0.93)$ & 106 (11) & $0.59(0.40-0.85)$ \\
\hline 3, 6A, 19A (13-valent) & 270 & 70 & $226(84)$ & 1 (Ref) & $20(7)$ & 1 (Ref) & $22(8)$ & 1 (Ref) & $199(74)$ & 1 (Ref) & $46(17)$ & 1 (Ref) \\
\hline 7 -valent vaccine & 988 & 64 & 806 (82) & $2.27(1.76-2.92)$ & $64(6)$ & $0.81(0.53-1.23)$ & $100(10)$ & $0.40(0.29-0.54)$ & $651(66)$ & $0.83(0.65-1.04)$ & 106 (11) & $0.76(0.55-1.06)$ \\
\hline Non-13-valent vaccine & 484 & 65 & $304(63)$ & 1 (Ref) & $38(8)$ & 1 (Ref) & $101(22)$ & 1 (Ref) & $339(70)$ & 1 (Ref) & $66(14)$ & 1 (Ref) \\
\hline $1,5,7 F$ (10-valent) & 354 & 53 & $316(90)$ & $4.33(2.92-6.40)$ & $13(4)$ & $0.45(0.23-0.85)^{\#}$ & 19 (5) & $0.20(0.12-0.34)$ & 132 (37) & $0.25(0.19-0.34)$ & $12(3)$ & $0.22(0.12-0.42)$ \\
\hline Non 13 -valent vaccine & 484 & 65 & $304(63)$ & 1 (Ref) & $38(8)$ & 1 (Ref) & $101(22)$ & 1 (Ref) & $339(70)$ & 1 (Ref) & $66(14)$ & 1 (Ref) \\
\hline 3, 6A, 19A (13-valent) & 270 & 70 & $226(84)$ & $2.60(1.79-3.79)$ & $20(7)$ & $0.93(0.53-1.64)$ & $22(8)$ & $0.31(0.19-0.51)$ & $199(74)$ & $1.20(0.86-1.67)$ & 46 (17) & $1.30(0.86-1.96)$ \\
\hline Non-13-valent vaccine & 484 & 65 & $304(63)$ & 1 (Ref) & $38(8)$ & 1 (Ref) & $101(22)$ & 1 (Ref) & $339(70)$ & 1 (Ref) & $66(14)$ & 1 (Ref) \\
\hline 13-valent vaccine & 1612 & 62 & $1348(84)$ & $2.62(2.07-3.31)$ & $97(6)$ & $0.75(0.51-1.11)$ & $141(9)$ & $0.34(0.26-0.45)$ & $982(61)$ & $0.67(0.54-0.83)$ & $164(10)$ & $0.72(0.53-0.97)^{\#}$ \\
\hline Non-13-valent vaccine & 484 & 65 & $304(63)$ & 1 (Ref) & $38(8)$ & 1 (Ref) & $101(22)$ & 1 (Ref) & $339(70)$ & 1 (Ref) & $66(14)$ & 1 (Ref) \\
\hline Total & 2096 & 63 & $1652(80)$ & & $135(6)$ & & 242 (12) & & 1321 (63) & & $230(11)$ & \\
\hline
\end{tabular}

Bold indicates statistical significance. Ref: reference. " : not significant after adjustment for multiple comparisons (Holm's method). 
TABLE 5 Clonal-specific disease manifestation and mortality in children in Sweden

\begin{tabular}{|c|c|c|c|c|c|c|c|c|c|c|c|}
\hline \multirow[t]{3}{*}{ Clonal complex } & \multirow[t]{3}{*}{ Serotypes } & \multirow{3}{*}{$\begin{array}{c}\text { Patients } \\
\text { n }\end{array}$} & \multirow{3}{*}{$\begin{array}{l}\text { Mean age } \\
\text { years }\end{array}$} & \multicolumn{5}{|c|}{ Disease manifestation ${ }^{\#}$} & \multirow{3}{*}{$\begin{array}{c}\begin{array}{c}\text { Any } \\
\text { underlying } \\
\text { disease }\end{array} \\
n(\%)\end{array}$} & \multirow{3}{*}{$\frac{\begin{array}{c}\text { 30-day } \\
\text { mortality" }\end{array}}{n(\%)}$} & \multirow{3}{*}{$\begin{array}{c}\text { ICU }>24 h^{7} \\
n(\%)\end{array}$} \\
\hline & & & & \multicolumn{2}{|c|}{ Pneumonia } & \multicolumn{2}{|c|}{ Meningitis } & \multirow{2}{*}{$\begin{array}{c}\text { Septicaemia }^{\text {१,+ }} \\
\mathrm{n}(\%)\end{array}$} & & & \\
\hline & & & & $\mathrm{n}(\%)$ & OR $(95 \%$ Cl) & n $(\%)$ & OR $(95 \% \mathrm{CI})$ & & & & \\
\hline 138 & $6 \mathrm{~B}^{f}, 4^{f}$ & 30 & 2 & $6(20)$ & $0.21(0.06-0.73)$ & $13(43)$ & $1.18(0.37-3.88)$ & $4(13)$ & $7(23)$ & 1 (3) & $9(32)$ \\
\hline 191 & $7 F^{\# \#}, 14^{f}$ & 29 & 5 & $16(55)$ & $0.99(0.31-3.15)$ & $7(24)$ & $0.51(0.15-1.77)$ & $4(14)$ & $6(21)$ & 0 & $2(7)$ \\
\hline 306 & $1^{\# \#}$ & 20 & 9 & 19 (95) & $10.52(1.96-108.98)$ & $0(0)$ & $0.04(0.00-0.35)$ & $1(5)$ & $1(5)$ & 0 & $3(15)$ \\
\hline 124 & $\begin{array}{c}14^{f}, 23 F^{f}, \\
19 A^{\text {ๆा }}\end{array}$ & 18 & 2 & $10(56)$ & 1 (Ref) & 7 (39) & 1 (Ref) & $1(6)$ & $5(28)$ & 0 & 7 (39) \\
\hline 9 & $14^{f}$ & 10 & 1 & $4(40)$ & $0.56(0.12-2.49)$ & $3(30)$ & $0.72(0.14-3.34)$ & $2(20)$ & $1(10)$ & 0 & $3(30)$ \\
\hline 156 & $9 \mathrm{Vf}^{f}$ & 10 & 4 & $4(40)$ & $0.56(0.12-2.49)$ & $1(10)$ & $0.24(0.02-1.42)$ & $2(20)$ & $3(30)$ & 0 & $1(10)$ \\
\hline 199 & $\begin{array}{c}19 A^{\text {กा }} \\
15 \mathrm{C}, 19 \mathrm{~F}^{f}\end{array}$ & 8 & 1 & 2 (25) & & $3(38)$ & & 2 (25) & $2(25)$ & 0 & $3(38)$ \\
\hline 113 & $18 \mathrm{C}^{f}, 18 \mathrm{~F}$ & 6 & 9 & $0(0)$ & & $2(40)$ & & $2(40)$ & 1 (20) & 0 & $1(20)$ \\
\hline 439 & $23 \mathrm{~F}^{f}$ & 5 & 6 & $1(20)$ & & $3(60)$ & & $0(0)$ & $2(40)$ & 0 & $1(20)$ \\
\hline 205 & $4^{f}$ & 5 & 6 & $2(40)$ & & $2(40)$ & & $1(20)$ & $1(20)$ & $1(20)$ & $1(20)$ \\
\hline 304 & $1^{\# \#}$ & 4 & 8 & $3(75)$ & & $1(25)$ & & $0(0)$ & $0(0)$ & 0 & $0(0)$ \\
\hline 180 & 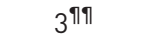 & 4 & 6 & $1(25)$ & & $2(50)$ & & $1(25)$ & $1(25)$ & $1(25)$ & $1(25)$ \\
\hline 53 & $8,11 \mathrm{~A}$ & 3 & 2 & $0(0)$ & & $3(100)$ & & $0(0)$ & $3(100)$ & 1 (33) & $2(67)$ \\
\hline 490 & $6 \mathrm{~A}^{\text {ตा }}$ & 3 & 1 & $0(0)$ & & $1(33)$ & & $2(67)$ & $2(67)$ & 0 & 1 (33) \\
\hline 460 & $35 \mathrm{~F}$ & 2 & 0 & $0(0)$ & & $0(0)$ & & $2(100)$ & $2(100)$ & 0 & $0(0)$ \\
\hline $\begin{array}{l}\text { Other } \mathrm{Cc}^{\S} \\
\text { vaccine types }\end{array}$ & & 28 & 4 & $10(36)$ & $0.44(0.13-1.44)$ & 9 (32) & $0.70(0.20-2.43)$ & $7(25)$ & $6(21)$ & 0 & $8(29)$ \\
\hline $\begin{array}{l}\text { Other } \mathrm{CC}^{\S} \\
\text { non-13-valent } \\
\text { vaccine types }\end{array}$ & & 7 & 5 & 2 (29) & & $3(43)$ & & 2 (29) & $4(57)$ & 0 & 2 (29) \\
\hline Total & & 192 & 4 & $80(42)$ & & 60 (31) & & $33(17)$ & $49(26)$ & $4(2)$ & 45 (23) \\
\hline
\end{tabular}

ICU: intensive care unit; CC: clonal complex; Ref: reference. ${ }^{\#}$ : patients can suffer from more than one manifestation; ${ }^{\text {I: }}$ not significant with ANOVA; ${ }^{+}$: septicaemia without a defined disease focus/manifestation; ${ }^{\S}$ : clonal complexes with <2 isolates, CC30, CC251, CC433 and predicted founder none; ${ }^{f}$ : serotypes included in the 7 -valent vaccine; ${ }^{\# \#}$ : additional serotypes

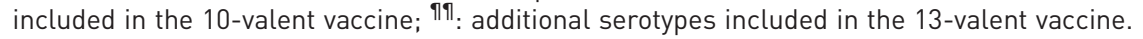




\begin{tabular}{|c|c|c|c|c|c|c|c|c|c|c|c|c|c|c|c|c|}
\hline \multirow{3}{*}{$\begin{array}{l}\text { Clonal } \\
\text { complex }\end{array}$} & \multirow[t]{3}{*}{ Serotypes" } & \multirow{3}{*}{$\begin{array}{l}\text { Patients } \\
\text { n }\end{array}$} & \multirow{3}{*}{$\begin{array}{c}\text { Mean age } \\
\text { years }\end{array}$} & \multicolumn{5}{|c|}{ Disease manifestation" } & \multirow{2}{*}{\multicolumn{2}{|c|}{ Mortality }} & \multicolumn{4}{|c|}{ Specific comorbidity } & \multicolumn{2}{|c|}{ Any underlying disease } \\
\hline & & & & \multicolumn{2}{|c|}{ Pneumonia } & \multirow{2}{*}{$\frac{\text { Meningitis }^{+}}{n(\%)}$} & \multicolumn{2}{|c|}{ Septicaemia ${ }^{\S}$} & & & \multirow{2}{*}{$\begin{array}{c}\begin{array}{c}\text { Cardiovascular } \\
\text { disease }\end{array} \\
\mathrm{n}(\%)\end{array}$} & \multirow{2}{*}{$\begin{array}{c}\begin{array}{c}\text { Pulmonary } \\
\text { disease }\end{array} \\
\mathrm{n}(\%)\end{array}$} & \multirow{2}{*}{$\begin{array}{c}\begin{array}{c}\text { Diabetes } \\
\text { mellitus }\end{array} \\
\mathrm{n}(\%)\end{array}$} & \multirow{2}{*}{$\frac{\text { Malignancy }}{n[\%]}$} & \multirow[b]{2}{*}{$n(\%)$} & \multirow[b]{2}{*}{ OR $(95 \% \mathrm{Cl})$} \\
\hline & & & & $\mathrm{n}(\%)$ & OR (95\% CI) & & $\mathrm{n}(\%)$ & OR $(95 \%$ Cl) & $n(\%)$ & OR $(95 \%$ Cl) & & & & & & \\
\hline 156 & $\begin{array}{c}9 V^{\# \#}, 14^{\# \#}, 9 A_{1} \\
19 F^{\# \#}, 24 \mathrm{~F}, 24 \mathrm{~A}, 15 \mathrm{~B}\end{array}$ & 217 & 61 & $180(83)$ & $0.75(0.41-1.33)$ & $14(6)$ & $22(10)$ & $1.19(0.60-2.43)$ & $21(10)$ & $1.32(0.65-2.78)$ & $54(26)$ & $38(19)$ & $22(10)$ & $26(13)$ & $130(60)$ & $0.87(0.56-1.35)$ \\
\hline 191 & $7 F^{\pi T}, 14^{\# \#}$ & 189 & 57 & $166(88)$ & $1.09(0.57-2.07)$ & $8(4)$ & $12(6)$ & $0.79(0.35-1.76)$ & $10(5)$ & $0.81(0.33-1.89)$ & $28(15)$ & $19(10)$ & $19(10)$ & 16 (9) & $83(44)$ & $0.50(0.32-0.78)$ \\
\hline 205 & $4^{\# \#}, 14^{\# \#}$ & 166 & 59 & $144(87)$ & $0.98(0.51-1.86)$ & $7(4)$ & $11(7)$ & $0.79(0.34-1.79)$ & $12(7)$ & $1.12(0.49-2.55)$ & $28(17)$ & $23(14)$ & $21(13)$ & $10(6)$ & $95(57)$ & $0.83(0.52-1.32)$ \\
\hline 124 & $\begin{array}{c}14^{\# \#}, 23 F^{\# \#}, 17 F, 8, \\
7 F^{\pi T}, 11 \mathrm{~A}\end{array}$ & 163 & 65 & $142(87)$ & 1 (Ref) & $10(6)$ & $15(9)$ & 1 (Ref) & $14(9)$ & 1 (Ref) & $42(27)$ & $28(18)$ & $16(10)$ & $32(20)$ & $108(66)$ & 1 (Ref) \\
\hline 306 & $1^{\pi \pi}$ & 126 & 50 & $118(94)$ & $2.28(0.99-5.71)$ & $4(3)$ & $2(2)$ & $0.21(0.03-0.79)$ & $3(2)$ & $0.48(0.11-1.54)$ & $12(10)$ & $3(2)$ & $11(9)$ & $4(3)$ & $41(33)$ & $0.38(0.22-0.63)$ \\
\hline 53 & $8,11 \mathrm{~A}, 11 \mathrm{C}$ & 93 & 64 & $70(75)$ & $0.45(0.23-0.87)$ & $9(10)$ & $13(14)$ & $1.56(0.69-3.46)$ & $15(16)$ & $2.00(0.90-4.48)$ & $24(26)$ & $27(30)$ & $15(16)$ & $14(15)$ & $65(70)$ & $1.27(0.72-2.26)$ \\
\hline 180 & $3^{++}$ & 93 & 69 & $82(88)$ & $1.08(0.50-2.42)$ & $7(8)$ & $4(4)$ & $0.43(0.12-1.24)$ & $15(16)$ & $1.94(0.87-4.33)$ & $36(41)$ & $22(24)$ & $14(16)$ & $9(10)$ & $65(70)$ & $1.06(0.60-1.88)$ \\
\hline 138 & $\begin{array}{c}6 B^{\# \#}, 6 \mathrm{~A}^{++}, 33 \mathrm{~F}, \\
23 \mathrm{~F}^{\# \#}\end{array}$ & 90 & 71 & $73(82)$ & $0.65(0.32-1.34)$ & $9(10)$ & $6(7)$ & $0.65(0.22-1.67)$ & $20(22)$ & $2.48(1.17-5.39)$ & $28(33)$ & $13(16)$ & $8(10)$ & $27(33)$ & $67(74)$ & $1.25(0.69-2.29)$ \\
\hline 439 & $23 F^{\# \#}, 6 \mathrm{~B}^{\# \#}$ & 84 & 71 & $65(78)$ & $0.54(0.27-1.09)$ & $6(7)$ & $10(12)$ & $1.21(0.50-2.82)$ & $12(14)$ & $1.52(0.65-3.51)$ & $30(38)$ & $20(26)$ & $12(15)$ & $19(23)$ & $62(74)$ & $1.19(0.66-2.20)$ \\
\hline 433 & $22 F, 23 F^{\# \#}$ & 76 & 67 & $56(74)$ & $0.42(0.21-0.83)$ & $7(9)$ & $14(18)$ & $2.16(0.97-4.79)$ & 5 (7) & $0.72(0.22-2.01)$ & $19(28)$ & $13(19)$ & $9(14)$ & $13(20)$ & $53(70)$ & $1.09(0.60-2.02)$ \\
\hline 66 & $9 N_{1} 9 \mathrm{~V}^{\# \#}, 16 \mathrm{~F}$ & 62 & 67 & $32(58)$ & $0.21(0.10-0.42)$ & $4(7)$ & $18(33)$ & $4.60(2.11-10.19)$ & $9(15)$ & $1.78(0.69-4.39)$ & $13(25)$ & $4(8)$ & $9(17)$ & $18(33)$ & $42(68)$ & $1.00(0.53-1.93)$ \\
\hline 9 & $14^{\# \#}, 19 F^{\# \#}, 12 \mathrm{~F}$, & 57 & 63 & $48(84)$ & $0.78(0.34-1.89)$ & $5(9)$ & $7(12)$ & $1.47(0.5-, 3.73)$ & $3(5)$ & $0.63(0.14-2.06)$ & $11(20)$ & $8(14)$ & $8(14)$ & $6(11)$ & $31(54)$ & $0.62(0.33-1.18)$ \\
\hline 199 & $19 \mathrm{~A}^{++}, 15 \mathrm{C}, 15 \mathrm{~B}, 10 \mathrm{~A}$ & 56 & 68 & $42(77)$ & $0.50(0.23-1.14)$ & 5 (9) & $5(9)$ & $0.92(0.29-2.52)$ & $9(16)$ & $1.78(0.69-4.42)$ & $16(30)$ & $11(21)$ & $8(15)$ & $14(26)$ & $44(79)$ & $1.73(0.85-3.74)$ \\
\hline 218 & $12 F, 22 F, 35 B, 19 V$ & 50 & 63 & $32(65)$ & $0.28(0.13-0.59)$ & $4(8)$ & $10(20)$ & $2.65(1.07-6.35)$ & $7(14)$ & $1.86(0.65-4.92)$ & $10(20)$ & $9(18)$ & $11(22)$ & $1(2)$ & $31(62)$ & $0.89(0.45-1.79)$ \\
\hline 490 & $6 \mathrm{~A}^{++}, 6 \mathrm{~B}^{\# \#}$ & 32 & 69 & $20(63)$ & $0.24(0.10-0.56)$ & $5(16)$ & $5(16)$ & $1.65(0.50-4.71)$ & $7(22)$ & $2.37(0.81-6.50)$ & $6(19)$ & $7(23)$ & $5(16)$ & $12(38)$ & $27(84)$ & $2.50(0.95-7.87)$ \\
\hline 113 & $18 C^{\# \#, 18 B}$ & 29 & 58 & $20(69)$ & $0.34(0.14-0.88)$ & $2(7)$ & $3(10)$ & $1.12(0.25-3.75)$ & $2(7)$ & $0.77(0.11-3.09)$ & $8(28)$ & $7(24)$ & $5(17)$ & $2(7)$ & $18(62)$ & $1.02(0.44-2.45)$ \\
\hline $100 / 496$ & $33 F_{1} 18 \mathrm{C}^{\# \#}, 23 \mathrm{~F}^{\# \#}$ & 25 & 63 & $16(64)$ & $0.26(0.10-0.69)$ & $1(4)$ & $7(28)$ & $3.83(1.30-10.57)$ & $2(8)$ & $0.88(0.13-3.56)$ & $3(13)$ & $6(25)$ & $4(17)$ & $4(17)$ & $19(76)$ & $1.87(0.71-5.56)$ \\
\hline $\begin{array}{l}\text { Other } \\
\text { cc }^{f}\end{array}$ & Vaccine types & 330 & 65 & $258(78)$ & $0.54(0.31-0.90)$ & $18(5)$ & $41(12)$ & $1.39(0.76-2.68)$ & $37(11)$ & $1.34(0.71-2.67)$ & $90(29)$ & $69(23)$ & 37 (12) & $52(17)$ & $226(68)$ & $1.14(0.75-1.73)$ \\
\hline $\begin{array}{l}\text { Other } \\
\text { cc }^{f}\end{array}$ & $\begin{array}{l}\text { Non-13-valent } \\
\text { vaccine types }\end{array}$ & 158 & 66 & $88(61)$ & $0.24(0.13-0.42)$ & $10(6)$ & $37(26)$ & $3.18(1.68-6.28)$ & $27(17)$ & $2.10(1.05-4.34)$ & $35(26)$ & $31(23)$ & $21(15)$ & $35(26)$ & $114(72)$ & $1.35(0.83-2.22)$ \\
\hline Total & & 2096 & 63 & $1652(80)$ & & $135(6)$ & $242(12)$ & & 230 (11) & & $493(25)$ & $358(18)$ & $255(13)$ & $314(16)$ & $1319(63)$ & \\
\hline
\end{tabular}

Odds ratios were adjusted for age and Charlson index score, calculated using patients with clinical data. Italic signifies the dominating serotype (>50\%). Bold indicates statistical

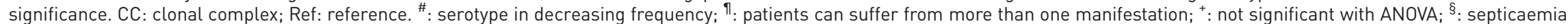
without a defined disease focus/manifestation; ${ }^{f}$ : CC with <25 isolates, CC460, CC304, CC568, CC260/378, CC414, CC72, CC251, CC309, CC30, CC3357, CC247, singletons and predicted founder none; ${ }^{\# \#}$ : serotypes included in the 7 -valent vaccine; ${ }^{\text {ศा }}$ : additional serotypes included in the 10 -valent vaccine; ${ }^{++}$: additional serotypes included in the 13 -valent vaccine. 
showed a lower odds ratio for pneumonia. Otherwise no significant changes were observed regarding clinical manifestations in children.

Among adults, fewer clonal complexes only expressed vaccine types (191, 205, 306, 180, 439 and 490) compared to in children (tables 5 and 6). In the largest clonal complex, CC156, four out of the seven serotypes were non-PCV13 serotypes. CC53, one of the more prevalent clonal complexes in adults, only expressed non-PCV13 capsules. Moreover, in this study we identified genetic lineages with significantly different disease manifestations. Thus, CC138, dominated by 6B but also including non-PCV13 serotypes in adults, showed a higher mortality. Septicaemia was found more often in patients with CC66, CC218 and CC100/496, where in each case the dominating serotype was a non-vaccine type. Of the less common clonal complexes, those including non-PCV types were associated with a higher odds ratio for septicaemia and a significantly higher mortality compared to reference ST124. However, in most cases there were no differences in age and odds ratio for specific comorbidities among the adult invasive pneumococcal disease patients depending on genetic lineage (table 6). Only CC306 (including only serotype 1) and CC191 (including types $7 \mathrm{~F}$ and 14) showed a lower odds ratio for causing disease in patients with previous underlying diseases.

\section{Discussion}

PCVs have shown protection against invasive pneumococcal disease caused by vaccine type pneumococci both in vaccinated and non-vaccinated individuals (due to herd immunity). However, these vaccines will inevitably promote expansion of non-vaccine type strains in the community. In the pre-vaccination material presented here, 23\% (484 out of 2096) of adult invasive pneumococcal disease patients were infected with non-PCV13 types consisting of 30 different serotypes. Thus, the serotype diversity found prior to vaccine introduction is sufficiently large to rapidly replace the diversity loss created by vaccine introduction in the community. Moreover, pre-existing non-vaccine type strains are capable of causing severe infections, but with a different spectrum of clinical manifestation compared to vaccine type strains.

By comparing invasive and carriage isolates among children from the same geographical area (Stockholm) we obtained an estimate of the attack rates provided by strains expressing different capsules [3, 29]. Our data suggest that non-PCV13 strains have a reduced invasive disease potential, which might result in lower incidence of invasive pneumococcal disease in the post-vaccination period, at least initially.

Furthermore, our analyses indicate that the spectrum of disease will change following type replacement with non-vaccine strains. The large size of the study with complete clinical records allows us to form a number of conclusions. In both children and adults, non-PCV13 strains had a reduced capacity to cause pneumonia and were more likely to infect patients with underlying diseases compared to strains with serotypes included in PCV7, PCV10 and PCV13. This is concordant with recent reports that non-PCV13 serotypes are more prevalent in children with comorbidities and immunocompromised adults [30, 31]. These findings are probably important reasons why non-PCV13 strains are such rare causes of invasive pneumococcal disease among children prior to the introduction of the vaccine. Importantly, non-PCV13 serotypes carried an increased risk of septicaemia with an unknown focus and tended to be associated with increased mortality in adults. Thus, although childhood vaccination with PCV will probably lead to an overall reduction of invasive pneumococcal disease, it will not lead to less severe pneumococcal disease among the remaining cases. Moreover, the clinical manifestations might be altered in non-vaccinated adults because of replacement with non-vaccine type strains due to herd immunity. So far, in our follow-up of the introduction of PCV in Sweden in 2009, we have not seen a decrease in the incidence of invasive pneumococcal disease among adults, even though we found a herd immunity effect with a reduced incidence of vaccine serotypes, but we also observed an increased incidence of non-vaccine type strains, in particular non-PCV13 serotypes (data not shown).

Prevalent pneumococcal strains belong to a number of clonal lineages (closely genetically related pneumococci as identified using molecular typing methods such as PFGE and MLST) of which some are successfully spread in the community [32]. Some of these lineages are associated with only one capsular serotype, but the majority may express several different capsules. By introducing PCV, depending on the capsular serotype expressed, some clonal lineages are expected to disappear, such as those expressing only vaccine serotypes. The most prevalent clonal complexes causing invasive pneumococcal disease in children prior to vaccine introduction in Sweden fall in this category. Replacement of these lineages with lineages expressing non-vaccine serotypes in the nasopharynx of healthy children will probably lead to a decreased incidence of invasive pneumococcal disease in children. For adults, the situation is different since genetic lineages that cause invasive pneumococcal disease seem to express non-vaccine type capsules more often. It is also worrisome that rare clonal lineages only expressing non-vaccine type capsules mediate a higher odds ratio of septicaemia and mortality compared to reference ST124. Thus, since it can be expected that these rare lineages will expand in the post-vaccination era we might witness a change in clinical 
manifestation of invasive pneumococcal disease in the adult population that will need further attention and new solutions such as novel vaccine approaches.

\section{Acknowledgements}

We would like to thank the clinical microbiological laboratories in Sweden, especially in Gothenburg, Skövde, Borås, Uddevalla, Halmstad and Stockholm, for providing pneumococcal isolates and Elisabeth Ek (Sahlgrenska University Hospital, Gothenburg), Berndt Claesson (Skaraborg Hospital, Skövde), Lars Johnsson (Borås Hospital, Borås), Gunilla Rådberg (Uddevalla Hospital, Uddevalla), and Torvald Ripa (Halmstad hospital, Halmstad, all Sweden) for collecting data and technical assistance. We also thank Staffan Normark (Karolinska Institutet, Stockholm, Sweden) for scientific input, and Ingrid Andersson, Gunnel Möllerberg and Christina Johansson (Swedish Institute for Infectious Disease Control, Solna, Sweden) for excellent technical expertise. Helle Bossen-Konradsen (Statens Serum Institut, Denmark) is greatly acknowledged for serotyping part of the isolates.

\section{References}

1 O'Brien KL, Wolfson LJ, Watt JP, et al. Burden of disease caused by Streptococcus pneumoniae in children younger than 5 years: global estimates. Lancet 2009; 374: 893-902.

2 Sjostrom K, Spindler C, Ortqvist A, et al. Clonal and capsular types decide whether pneumococci will act as a primary or opportunistic pathogen. Clin Infect Dis 2006; 42: 451-459.

3 Sandgren A, Sjostrom K, Olsson-Liljequist B, et al. Effect of clonal and serotype-specific properties on the invasive capacity of Streptococcus pneumoniae. J Infect Dis 2004; 189: 785-796.

4 Sandgren A, Albiger B, Orihuela CJ, et al. Virulence in mice of pneumococcal clonal types with known invasive disease potential in humans. J Infect Dis 2005; 192: 791-800.

5 Harboe ZB, Thomsen RW, Riis A, et al. Pneumococcal serotypes and mortality following invasive pneumococcal disease: a population-based cohort study. PLoS Med 2009; 6: e1000081.

6 Whitney CG, Farley MM, Hadler J, et al. Decline in invasive pneumococcal disease after the introduction of protein-polysaccharide conjugate vaccine. N Engl J Med 2003; 348: 1737-1746.

7 Park SY, Van Beneden CA, Pilishvili T, et al. Invasive pneumococcal infections among vaccinated children in the United States. J Pediatr 2010; 156: 478-483.

8 Pilishvili T, Lexau C, Farley MM, et al. Sustained reductions in invasive pneumococcal disease in the era of conjugate vaccine. J Infect Dis 2010; 201: 32-41.

9 Rodenburg GD, de Greeff SC, Jansen AG, et al. Effects of pneumococcal conjugate vaccine 2 years after its introduction, the Netherlands. Emerg Infect Dis 2010; 16: 816-823.

10 Foster D, Walker AS, Paul J, et al. Reduction in invasive pneumococcal disease following implementation of the conjugate vaccine in the Oxfordshire region, England. J Med Microbiol 2011; 60: 91-97.

11 van der Linden M, Weiss S, Falkenhorst G, et al. Four years of universal pneumococcal conjugate infant vaccination in Germany: impact on incidence of invasive pneumococcal disease and serotype distribution in children. Vaccine 2012; 30: 5880-5885.

12 Durando P, Faust SN, Fletcher $\mathrm{M}$, et al. Experience with pneumococcal polysaccharide conjugate vaccine (conjugated to CRM197 carrier protein) in children and adults. Clin Microbiol Infect 2013; 19: Suppl. 1, 1-9.

13 Sherwin RL, Gray S, Alexander R, et al. Distribution of 13-valent pneumococcal conjugate vaccine Streptococcus pneumoniae serotypes in US adults aged $\geqslant 50$ years with community-acquired pneumonia. J Infect Dis 2013; 208 : $1813-1820$.

14 Cohen R, Levy C, Bonnet E, et al. Risk factors for serotype 19A carriage after introduction of 7-valent pneumococcal vaccination. BMC Infect Dis 2011; 11: 95.

15 Kaplan SL, Barson WJ, Lin PL, et al. Serotype 19A Is the most common serotype causing invasive pneumococcal infections in children. Pediatrics 2010; 125: 429-436.

16 Henriques B, Kalin M, Ortqvist A, et al. Molecular epidemiology of Streptococcus pneumoniae causing invasive disease in 5 countries. J Infect Dis 2000; 182: 833-839.

17 Lexau CA, Lynfield R, Danila R, et al. Changing epidemiology of invasive pneumococcal disease among older adults in the era of pediatric pneumococcal conjugate vaccine. JAMA 2005; 294: 2043-2051.

18 Griffin MR, Zhu Y, Moore MR, et al. U.S. hospitalizations for pneumonia after a decade of pneumococcal vaccination. $N$ Engl J Med 2013; 369: 155-163.

19 Feikin DR, Kagucia EW, Loo JD, et al. Serotype-specific changes in invasive pneumococcal disease after pneumococcal conjugate vaccine introduction: a pooled analysis of multiple surveillance sites. PLoS Med 2013; 10: e1001517.

20 Alanee SR, McGee L, Jackson D, et al. Association of serotypes of Streptococcus pneumoniae with disease severity and outcome in adults: an international study. Clin Infect Dis 2007; 45: 46-51.

21 Berg S, Trollfors B, Persson E, et al. Serotypes of Streptococcus pneumoniae isolated from blood and cerebrospinal fluid related to vaccine serotypes and to clinical characteristics. Scand J Infect Dis 2006; 38: 427-432.

22 Browall S, Norman M, Tangrot J, et al. Intraclonal variations among Streptococcus pneumoniae isolates influence the likelihood of invasive disease in children. J Infect Dis 2014; 209: 377-388.

23 Henriqus Normark B, Christensson B, Sandgren A, et al. Clonal analysis of Streptococcus pneumoniae nonsusceptible to penicillin at day-care centers with index cases, in a region with low incidence of resistance: emergence of an invasive type 35B clone among carriers. Microb Drug Resist 2003; 9: 337-344.

24 Trollfors B, Berg S, Backhaus E, et al. Invasive, paediatric, vaccine strains of Streptococcus pneumoniae: are there differences in clinical characteristics? Scand J Infect Dis 2009; 41: 84-87.

25 Hermans PW, Sluijter M, Hoogenboezem T, et al. Comparative study of five different DNA fingerprint techniques for molecular typing of Streptococcus pneumoniae strains. J Clin Microbiol 1995; 33: 1606-1612.

26 Enright MC, Spratt BG. A multilocus sequence typing scheme for Streptococcus pneumoniae: identification of clones associated with serious invasive disease. Microbiology 1998; 144: 3049-3060.

27 Holm S. A simple sequentially rejective multiple test procedure. Scand J Statist 1979; 6: 65-70.

28 Firth D. Bias reduction of maximum likelihood estimates. Biometrika 1993; 80: 27-38. 
29 Brueggemann AB, Griffiths DT, Meats E, et al. Clonal relationships between invasive and carriage Streptococcus pneumoniae and serotype- and clone-specific differences in invasive disease potential. J Infect Dis 2003; 187: $1424-1432$.

30 Ladhani SN, Slack MP, Andrews NJ, et al. Invasive pneumococcal disease after routine pneumococcal conjugate vaccination in children, England and Wales. Emerg Infect Dis 2013; 19: 61-68.

31 Lujan M, Burgos J, Gallego M, et al. Effects of immunocompromise and comorbidities on pneumococcal serotypes causing invasive respiratory infection in adults: implications for vaccine strategies. Clin Infect Dis 2013; 57: $1722-1730$.

32 Sjostrom K, Blomberg C, Fernebro J, et al. Clonal success of piliated penicillin nonsusceptible pneumococci. Proc Natl Acad Sci USA 2007; 104: 12907-12912. 